\title{
SYMMETRIC BEHAVIOR IN FUNCTIONS
}

\author{
UDAYAN B. DARJI
}

(Communicated by Andrew M. Bruckner)

\begin{abstract}
S. Marcus raised the following problem: Find necessary and sufficient conditions for a set to be the set of points of symmetric continuity of some function $f: R \rightarrow R$. We show that there is no such characterization of topological nature. We prove that given a zero-dimensional set $M \subseteq R$, there exists a function $f: R \rightarrow R$ whose set of points of symmetric continuity is topologically equivalent to $M$. Thus, there is no "upper bound" on the topological complexities of $M$. We also prove similar theorems about the set of points where a function may be symmetrically differentiable, symmetric, or smooth.
\end{abstract}

\section{INTRODUCTION}

Let $f: R \rightarrow R$. Then $f$ is said to be smooth at a point $p$ if

$$
\lim _{h \rightarrow 0} \frac{f(p+h)+f(p-h)-2 f(p)}{2 h}=0,
$$

and $f$ is symmetric at $p$ if

$$
\lim _{h \rightarrow 0} f(p+h)+f(p-h)-2 f(p)=0 .
$$

We say that $f^{s}(p)$ is the symmetric derivative of $f$ at $p$ if

$$
\lim _{h \rightarrow 0} \frac{f(p+h)-f(p-h)}{2 h}=f^{s}(p),
$$

and $p$ is a point of symmetric continuity of $f$ if

$$
\lim _{h \rightarrow 0} f(p+h)-f(p-h)=0 .
$$

If $f$ is continuous at $p$, then $f$ is symmetric at $p$ and $p$ is also a point of symmetric continuity of $f$. If $f$ is differentiable at $p$, then $f$ is smooth at $p$ and the derivative of $f$ is also the symmetric derivative of $f$ at $p$. If a function is smooth at a point or symmetric at a point, then we know that the

Received by the editors March 24, 1992; this paper was presented at the 8th Auburn Miniconference on Real Analysis, sponsored by NSF.

1991 Mathematics Subject Classification. Primary 26A21, 26A15; Secondary 54C30, 54C50, $54 \mathrm{H} 05$.

Key words and phrases. Symmetric continuity, symmetric derivative, smooth, perfect linearly independent sets, the Baire property, Borel functions, coanalytic sets. 
"odd" behavior of the function is nice at that point. On the other hand, if a function has a symmetric derivative at a point or the function is symmetrically continuous at a point, then we know that the "even" behavior of the function is nice at that point. If $f$ is a function, then $\operatorname{SM}(f), \operatorname{SD}(f), \mathbf{S}(f)$, and $\operatorname{SC}(f)$ denotes the set of all points where $f$ is smooth, symmetrically differentiable, symmetric, and symmetrically continuous, respectively. The reader may refer to [3] for further historical information on the subject.

If $r \in R$ and $A, B \subseteq R$, then $r A=\{r x$ : such that $x \in A\}$ and $A+B=$ $\{x+y: x \in A$ and $y \in B\}$. By $A-B$ we mean $A+(-1) B$.

Marcus submitted the following problem at the Summer Symposium on Real Analysis at Smolenice, Czechoslovakia, August, 1991: Given an arbitrary real function, characterize its points of symmetric continuity. Apparently he had given some thought to this problem in the 50s. In [5] he shows that given an arbitrary $F_{\sigma}$ set $M$, there exists a function whose points of symmetric continuity is exactly $M^{\mathrm{c}}$. In this paper we prove the following theorem: If $M$ is a zero-dimensional subset of $R$, then there exists a function $f$ which is Lebesgue measurable and has the Baire property such that $\operatorname{SC}(f)$ is homeomorphic to $M$. This theorem tells us that there is not even an "upper bound" on the topological complexities of $\operatorname{SC}(f)$ for an arbitrary function $f$.

When the author presented his argument for this result to the analysis seminar at North Carolina State University, Mike Evans asked him if he could modify his argument to get similar results for the differentiable aspects of symmetric behavior. The author was able to do this. In fact, given a zero-dimensional subset of $R$, we will construct a Lebesgue measurable function $f: R \rightarrow R$ with the Baire property such that $\mathrm{SM}(f)$ is homeomorphic to $M$ and $f$ is not symmetric on $R \backslash \operatorname{SM}(f)$. The absolute value of this function will have the property that $\operatorname{SD}(|f|)$ is homeomorphic to $M$ and $|f|$ is not symmetrically continuous at any point of $R \backslash \mathrm{SD}(f)$. As a consequence we have that there is no "upper bound" on the topological complexities of $\mathbf{S M}(f), \operatorname{SD}(f), \mathbf{S}(f)$, and $\operatorname{SC}(f)$ for an arbitrary function $f$.

However, if we have more information about $f$, then we can say something additional about $\mathrm{S}(f)$ and $\mathrm{SC}(f)$. In Theorem 1 we show that if $f$ is Borel, then $\mathrm{S}(f)$ and $\mathrm{SC}(f)$ have to be coanalytic. And, if $f$ is of Baire class 1 , then we have that $\mathrm{S}(f)$ and $\mathrm{SC}(f)$ are $G_{\delta \sigma \delta}$.

It is easy to show that if $G \subseteq R$ is a nontrivial linear space over $Q$, then $\operatorname{SD}\left(\chi_{G}\right)=\operatorname{SC}\left(\chi_{G}\right)=G$, where $\chi_{G}$ denotes the characteristic function of $G$.

\section{MAIN RESULtS}

Theorem 1. Suppose $f: R \rightarrow R$. Then we have the following.

(1) If $f$ is Lebesgue measurable, then $\mathrm{S}(f)$ and $\mathrm{SC}(f)$ are Lebesgue measurable.

(2) If $f$ has the Baire property, then $\mathrm{S}(f)$ and $\mathrm{SC}(f)$ have the Baire property.

(3) If $f$ is of Baire class one, then $\mathrm{S}(f)$ and $\mathrm{SC}(f)$ are $G_{\delta \sigma \delta}$. If $f$ is Borel measurable, then $S(f)$ and $\mathrm{SC}(f)$ are coanalytic.

Proof. In [1] the author proves (Corollaries 2 and 4) that if $f$ is Lebesgue measurable or has the Baire property, then $\mathbf{S}(f)$ is of measure zero or of the 
first category, respectively. These arguments with very minor modifications yield similar results about $\operatorname{SC}(f)$.

Now let us show the third part of the theorem. First, we will show that if $f$ is of Baire class one, then $\operatorname{SC}(f)$ is $G_{\delta \sigma \delta}$ and if $f$ is Borel then $\operatorname{SC}(f)$ is coanalytic. For each positive integer $m$, let $B_{m} \equiv\{I \subseteq R: I$ is an open interval with rational end points and the length of $I$ is less than $1 / m\}$. For each positive integer $n$, rational $r$, and $I \in B_{m}$, let

$$
A(n, r, I)=\left[\left(f^{-1}(-\infty, r) \cap I\right)+\left(f^{-1}(r+1 / n, \infty) \cap I\right)\right] / 2 .
$$

Now we claim that $R \backslash \mathrm{SC}(f)=\bigcup_{n=1}^{\infty} \bigcap_{m=1}^{\infty} \bigcup_{I \in B_{m}} \bigcup_{r \in Q} A(n, r, I)$. To see this consider the following argument: Let $x \in R \backslash \mathrm{SC}(f)$. Let $k$ be a positive integer such that for every $\delta>0$, there is $0<h<\delta$ such that $|f(x+h)-f(x-h)|>$ $2 / k$. Let $m$ be a positive integer. There is $I \in B_{m}$ such that $x \in I$. Let $h$ be such that $x+h$ and $x-h$ are in $I$, and $|f(x+h)-f(x-h)|>2 / k$. Let $r$ be a rational number such that $r$ and $r+1 / k$ are between $f(x+h)$ and $f(x-h)$. Then $x \in A(k, r, I)$. Since $m$ was chosen arbitrarily, we have that $x \in$ $\bigcap_{m=1}^{\infty} \bigcup_{I \in B_{m}} \bigcup_{r \in Q} A(k, r, I) \Rightarrow x \in \bigcup_{n=1}^{\infty} \bigcap_{m=1}^{\infty} \bigcup_{I \in B_{m}} \bigcup_{r \in Q} A(n, r, I)$. That $x \in \bigcup_{n=1}^{\infty} \bigcap_{m=1}^{\infty} \bigcup_{I \in B_{m}} \bigcup_{r \in Q} A(n, r, I) \Rightarrow x \in R \backslash \mathrm{SC}(f)$ is easy to check.

If $f$ is of Baire class one, then $A(n, r, I)$ is $F_{\sigma}$ because the sum of two $F_{\sigma}$ sets is $F_{\sigma}$. Since $R \backslash \mathrm{SC}(f)$ is the countable union of the countable intersection of $F_{\sigma}$ sets, $R \backslash \mathrm{SC}(f)$ is $F_{\sigma \delta \sigma}$, which, in turn, implies that $\mathrm{SC}(f)$ is $G_{\delta \sigma \delta}$.

If $f$ is Borel, then we have a different situation. Erdös and Stone in [2] show that there exists a $G_{\delta}$ subset of the line and a compact subset of the line whose sum is not a Borel set. Another similar result is that of Rogers [8], where he constructs a $G_{\delta}$ subset of the line whose difference set is not Borel. Hence we cannot say that $A(n, r, I)$ is Borel in general. However, we know that $A(n, r, I)$ is analytic because it can be viewed as a continuous image of a Borel subset of the plane. Further, since the countable union and the countable intersection of analytic sets is analytic, we get that $R \backslash \mathrm{SC}(f)$ is analytic, which, in turn, implies that $\mathrm{SC}(f)$ is coanalytic.

Now we want to show that if $f$ is Borel then $\mathbf{S}(f)$ is coanalytic and if $f$ is of Baire class one then $\mathrm{S}(f)$ is $G_{\delta \sigma \delta}$.

For each positive integer $m$, let $B_{m}$ be as before. For each positive integer $n$, and for $(r, s),(t, u), I \in B_{m}$, let

$$
\begin{aligned}
A(n,(r, s),(t, u), I)= & {\left[\left(f^{-1}((r, s)) \cap I\right)+\left(f^{-1}((t, u)) \cap I\right)\right] / 2 } \\
& \cap f^{-1}\left(R \backslash\left[-n^{-1}+(r+t) / 2, n^{-1}+(s+u) / 2\right]\right) .
\end{aligned}
$$

First, we claim that

$$
R \backslash \mathrm{S}(f)=\bigcup_{n=1}^{\infty} \bigcap_{m=1}^{\infty} \bigcup_{J \in B_{m}} \bigcup_{K \in B_{m}} \bigcup_{I \in B_{m}} A(n, J, K, I) .
$$

To see this consider the following argument: Let $x \in R \backslash \mathbf{S}(f)$. Let $n$ be a positive integer such that for every $\delta>0$, there is $0<h<\delta$ such that $|f(x+h)+f(x-h)-2 f(x)|>5 / n$. Let $m$ be a positive integer. There is $I \in B_{m}$ such that $x \in I$. Let $h$ be such that $x+h$ and $x-h$ are in $I$, and $|f(x+h)+f(x-h)-2 f(x)|>5 / n$. Let $J, K \in B_{m}$ be such that $f(x+h)$ is in $J, f(x-h)$ is in $K$, and the lengths of each of $J$ and $K$ is less than 
$1 / n$. Then $x \in A(n, J, K, I)$. Since $m$ was chosen arbitrarily, we have that

$$
\begin{aligned}
x \in & \bigcap_{m=1}^{\infty} \bigcup_{J \in B_{m}} \bigcup_{K \in B_{m}} \bigcup_{I \in B_{m}} A(n, J, K, I) \\
& \Rightarrow x \in \bigcup_{n=1}^{\infty} \bigcap_{m=1}^{\infty} \bigcup_{J \in B_{m}} \bigcup_{K \in B_{m}} \bigcup_{I \in B_{m}} A(n, J, K, I) .
\end{aligned}
$$

That $x \in \bigcup_{n=1}^{\infty} \bigcap_{m=1}^{\infty} \bigcup_{J \in B_{m}} \bigcup_{K \in B_{m}} \bigcup_{I \in B_{m}} A(n, J, K, I) \Rightarrow x \in R \backslash \mathrm{S}(f)$ is easy to check.

As previously, if $f$ is of Baire class one, then $A(n, J, K, I)$ is $F_{\sigma}$. Since $R \backslash \mathbf{S}(f)$ is the countable union of the countable intersection of $F_{\sigma}$ sets, $R \backslash \mathbf{S}(f)$ is $F_{\sigma \delta \sigma}$, which, in turn, implies that $\mathrm{S}(f)$ is $G_{\delta \sigma \delta}$.

Again, as before, if $f$ is Borel then $A(n, J, K, I)$ is analytic. Since $R \backslash \mathrm{S}(f)$ is the countable union of the countable intersection of analytic sets, $R \backslash \mathrm{S}(f)$ is analytic, which, in turn, implies that $\mathbf{S}(f)$ is coanalytic.

The reader may refer to [1] for some examples of functions which are pathological with respect to symmetric behavior.

Theorem 2. Let $M$ be a zero-dimensional subset of $R$. Then there are functions $f_{1}, f_{2}, f_{3}, f_{4}$ such that $M$ is topologically equivalent to $\mathrm{S}\left(f_{1}\right), \operatorname{SC}\left(f_{2}\right), \operatorname{SD}\left(f_{3}\right)$, and $\operatorname{SM}\left(f_{4}\right)$.

Proof. Given a zero-dimensional set $M$, we will prove that there exists a function $f$ such that $\operatorname{SM}(f)$ is homeomorphic to $M$ and $f$ is not symmetric at any point of $R \backslash \operatorname{SM}(f)$ as well. We will construct this function carefully enough so that the function $g \equiv|f|$ will be such that $\operatorname{SD}(g)$ is homeomorphic to $M$ and $g$ is not symmetrically continuous at any point of $R \backslash \mathrm{SD}(g)$ as well. This will prove all the claims that are made in the theorem.

In our proof we use the fact that there is a Cantor set which is linearly independent over $Q$, the set of rational numbers. The existence of such a Cantor set follows from an old result of von Neumann [7]. Mycielski [6] has shown, among more general results, that every connected, locally compact topological group with a complete metric contains a linearly independent Cantor set. We will not construct such a Cantor set here, but the reader may refer to [4] for a simple construction of a linearly independent Cantor set.

Let us begin our proof of the theorem. Let $M$ be a zero-dimensional subset of $R$. Let $C_{1}$ be a Cantor set, and let $E_{1}, E_{2}, E_{3}, \ldots$ be pairwise disjoint countable dense subsets of $R \backslash C_{1}$ such that $C \equiv C_{1} \cup E_{1} \cup E_{1} \cup E_{2} \cup E_{3} \ldots$ is linearly independent over $Q$, the set of rational numbers. Let $G$ be the group generated by the set of all finite linear combinations of $C$. That $G$ is of the first category and measure zero follows by an argument similar to that of Lemma 10 in [1]. Let $A$ be a proper subset of $C_{1}$ such that $A$ is homeomorphic to $M$, and let $B=C_{1} \backslash A$. Note that $A, B$ and $E_{1}, E_{2}, E_{3}, \ldots$ are disjoint and that each of $E_{1}, E_{2}, E_{3}, \ldots$ is dense in $R$.

Throughout the following construction, $\sigma, \tau, \zeta, \psi, \pi$ will be used for finite sequences of 0 's and 1's. If $\sigma$ is a finite sequence of length $n \geq m$, then $\sigma \mid m$ will denote the first $m$-term truncation of $\sigma$ and $\sigma \mid 0$ will denote $\varnothing$.

For each $\sigma$ we now define the set $L_{\sigma}$ in the following manner: Define $L_{\varnothing} \equiv B$. Let $L_{0} \equiv\left[2 A-L_{\varnothing}\right] \backslash L_{\varnothing}$ and $L_{1} \equiv\left[2 L_{\varnothing}-E_{1}\right] \backslash L_{\varnothing}$. We now 
induct on the length of $\sigma$. Suppose, for positive integer $n \geq 1, L_{\sigma}$ is defined for all sequences $\sigma$ of length $\leq n$. If $\tau$ is a sequence of length $n+1$, let $L_{\tau} \equiv\left[2 A-L_{\tau \mid n}\right] \backslash\left(\bigcup_{\sigma:|\sigma| \leq n} L_{\sigma}\right)$, provided that the last term of $\tau$ is 0 ; otherwise, let $L_{\tau} \equiv\left[2 L_{\tau \mid n}-E_{j}\right] \backslash\left(\bigcup_{\sigma:|\sigma| \leq n} L_{\sigma}\right)$ where $j$ is the least positive integer which has not been used in the construction of any of the previous $L$ 's. The order in which the $L_{\tau}$ are defined will not make any difference as long as $j$ is picked in the prescribed manner.

Each $L_{\sigma}$ is a subset of $G$, so each element of $L_{\sigma}$ has a unique linear representation in terms of $C$. In what follows, all linear representations are assumed to be in terms of $C$ and they are assumed to be unique, which implies that no element of $C$ appears twice in a representation.

We now state some lemmas. As the proofs of these lemmas are somewhat technical, they will not be proved until later so as not to obscure the overall picture of the argument.

Lemma 1. Suppose that $\sigma \neq \tau$, and $|\sigma|=|\tau|$. Then there is a positive integer $i$ such that (1) for all $x \in L_{\sigma}$ the linear representation of $x$ contains some element of $E_{i}$ and for all $y \in L_{\tau}$ the linear representation of $y$ does not contain any element of $E_{i}$, or (2) for all $y \in L_{\tau}$ the linear representation of $y$ contains some element of $E_{i}$, and for all $x \in L_{\sigma}$ the linear representation of $x$ does not contain any element of $E_{i}$. As a consequence, we have that if $\sigma \neq \tau$, then $L_{\sigma} \cap L_{\tau}=\varnothing$.

Lemma 2. If $x \in A$ and $y \in L_{\sigma}$, then there is $\tau$ such that (1) $2 x-y \in L_{\tau}$, (2) ||$\sigma|-| \tau||=1$, and (3) either $\sigma$ is an extension of $\tau$ and the last term of $\sigma$ is zero or $\tau$ is an extension of $\sigma$ and the last term of $\tau$ is zero.

Lemma 3. Let $M$ be a nowhere dense subset of an open set $U$. Let $N \subseteq U$ such that $N$ is c-dense in $U$ and $M \cap N=\varnothing$. Then there is a set $K \subseteq N$ such that for every $x \in M$ there is a sequence $\left\{x_{n}\right\} \rightarrow x$ such that, for every $n$, $x_{n} \in K$ and $2 x-x_{n} \notin M \cup K$.

First we will define a function $h: R \rightarrow R$ which is smooth at every point of $A$ but not symmetric at any point of the $L_{\sigma}$ 's. Then we will modify this function using Lemma 3 to get our final function $f$ which is smooth at every point of $A$ but not symmetric anywhere else.

We define $h: R \rightarrow R$ inductively. Let $h\left(L_{\varnothing}\right)=2$. Suppose $h\left(L_{\sigma}\right)$ has been defined for all $\sigma$ such that $|\sigma| \leq n$. If $\tau$ is a sequence such that $|\tau|=n+1$, then define $h\left(L_{\tau}\right)=-h\left(L_{\tau \mid n}\right)$ provided that the last term of $\tau$ is zero; otherwise, let $h\left(L_{\tau}\right)=1+h\left(L_{\tau \mid n}\right)$ if $h\left(L_{\tau \mid n}\right)$ is positive and $h\left(L_{\tau}\right)=-1+h\left(L_{\tau \mid n}\right)$ if $h\left(L_{\tau \mid n}\right)$ is negative. Let $h$ be zero on the complement of $\bigcup L_{\sigma}$. In particular, note that $h$ is zero on $A$ and $E_{1}, E_{2}, E_{3}, \ldots$. That $h$ is well defined follows from Lemma 1 . Also note that $h$ only takes integer values bigger than 1 or less than -1 on $L_{\sigma}$ 's.

We now want to show that $h$ is smooth at every point of $A$. Let $x \in A$. If $y \in L_{\sigma}$ for some $\sigma$, then, by Lemma 2, there is $\tau$ such that (1) $2 x-y \in L_{\tau}$, (2) ||$\sigma|-| \tau||=1$, and (3) either $\sigma$ is an extension of $\tau$ and the last term of $\sigma$ is zero or $\tau$ is an extension of $\sigma$ and the last term of $\tau$ is zero. But, by properties 2 and 3 , and by the method in which $h$ was defined, we have that $h(y)=-1 h(2 x-y)$. Since $h(x)=0$, we have that $|h(y)+h(2 x-y)-2 h(x)|=$ 0 . If $y \notin L_{\sigma}$ for any $\sigma$, then $h(y)=h(2 x-y)=0$ because both $y$ and 
$2 x-y$ belong to some $L \zeta$ or neither one of them does. In this case we also have that $|h(y)+h(2 x-y)-2 h(x)|=0$.

We now want to show that $h$ is not symmetric at each point of the $L_{\sigma}$ 's. Let $p \in L_{\sigma}$ for some $\sigma$. Let $\zeta$ be such that $|\zeta|=|\sigma|+1$, and the last term of $\zeta$ is 1 . Then $L_{\zeta}=\left[2 L_{\sigma}-E_{j}\right] \backslash\left(\bigcup_{\psi:|\psi| \leq|\sigma|} L_{\psi}\right)$, where $j$ is a positive integer which has not been used previously in the construction of $L$ 's. Recall that $E_{j}$ is dense in $R$, so there exists a sequence $\left\{z_{n}\right\}_{n=1}^{\infty}$ in $E_{j}$ which converges to $p$. Because each $2 p-z_{n}$ belongs to $L_{\zeta}, h$ is zero on $E_{j}$, and the way $h$ was defined, we have that

$$
\left|h\left(2 p-z_{n}\right)+h\left(z_{n}\right)-2 h(p)\right|=\left|h\left(2 p-z_{n}\right)-2 h(p)\right| \geq 1 .
$$

Therefore, $h$ is not symmetric at $p$. In particular, we have that $h$ is not symmetric at any point of $B$ because $L_{\varnothing}=B$.

That $h$ is not continuous at any point follows from the fact that $h$ is zero on the dense set $G^{\mathrm{c}}$ and $h$ is 3 on the dense set $L_{1}$.

Using arguments similar to the above, the reader can verify that $|h|$ is symmetrically differentiable at every point of $A$ with symmetric derivative zero and that $|h|$ is not symmetrically continuous at any point of $L_{\sigma}$. It is also the case that $|h|$ is not continuous anywhere.

Our functions $h$ and $|h|$ are Lebesgue measurable and have the Baire property because each of $h$ and $|h|$ are zero on a residual full measure set, namely, $G^{\mathrm{c}}$. By Theorems 1 and 3 in [1], $S(h) \backslash C(h)$ has to be of the first category and measure zero. With very minor modifications of those theorems, it also follows that $\mathrm{SC}(|h|) \backslash C(\mid h) \mid)$ have to be of the first category and measure zero. Since neither $h$ nor $|h|$ is continuous anywhere, we have that $\mathrm{S}(h) \cup \mathrm{SC}(|h|)$ is of the first category and measure zero. Since $B=C_{1} \backslash A, h$ is not symmetric at any point of $B$, and $|h|$ is not symmetrically continuous at any point of $B$, we have that $[\mathrm{S}(h) \cup \mathrm{SC}(|h|)] \backslash A \cap C_{1}=\varnothing$.

Let $\left\{F_{n}\right\}_{n=1}^{\infty}$ be a sequence of nowhere dense sets such that (1) if $i \neq j$ then $F_{i} \cap F_{j}=\varnothing$, (2) $\mathrm{Cl}\left(F_{n}\right) \cap C_{1}=\varnothing$, and (3) $\bigcup_{n=1}^{\infty} F_{n}=[\mathrm{S}(h) \cup \mathrm{SC}(|h|)] \backslash A$. Let $\left\{U_{n}\right\}_{n=1}^{\infty}$ and $\left\{V_{n}\right\}_{n=1}^{\infty}$ be sequences of open sets such that $F_{n} \subseteq U_{n}, C_{1} \subseteq V_{n}$, $U_{n} \cap V_{n}=\varnothing, \operatorname{cl}\left(V_{n+1}\right) \subseteq V_{n}$, and the distance from $C_{1}$ to $V_{n}^{\text {c }}$ is less than $1 /(n+2)$. For each positive integer $n$, let $\varepsilon_{n}$ be the distance from $C_{1}$ to $V_{n}^{\mathrm{c}}$. Since $c l\left(V_{n+1}\right) \subseteq V_{n}$ and the distance from $C_{1}$ to $V_{n}^{\mathrm{c}}$ is less than $1 /(n+2)$, we have that $\left\{\varepsilon_{n}\right\}$ is a decreasing sequence between 0 and $1 / 3$. Let $\left\{D_{n}\right\}_{n=1}^{\infty}$ be a sequence of dense subsets of $G^{\mathrm{c}}$ such that $D_{n}$ is $c$-dense in $U_{n}, D_{n}$ is of the first category and measure zero, $D_{n} \cap F_{n}=\varnothing$, and if $i \neq j$, then $D_{i} \cap D_{j}=\varnothing$. By Lemma 3, obtain $K_{n} \subseteq D_{n}$ such that for every $x \in F_{n}$, there is a sequence $\left\{x_{n}\right\} \rightarrow x$ such that, for every $n, x_{n} \in K$ and $2 x-x_{n} \notin F_{n} \cup K_{n}$. Now define $f$ in the following manner. If $x \in F_{n} \cup K_{n}$ then $f(x)=\varepsilon_{n}^{2}$; otherwise let $f(x)=h(x)$. If $p \notin L_{\sigma}$ for any $\sigma$, then $f(x)$ is between 0 and $1 / 9$.

We now claim that $f$ is smooth at every point of $A$. Let $x \in A$. Let $\varepsilon>0$, and let $n$ be such that $\varepsilon_{n}<\varepsilon$. Let $\delta$ be such that the interval centered at $x$ of length $\delta$ is contained in $V_{n}$. Let $y$ be such that $|x-y|<\delta$. If $y \in L_{\sigma}$ for some $\sigma$, then as before we have that $|f(y)+f(2 x-y)-2 f(x)|=0$ because $f$ is the same as $h$ on $A$ and $L$ 's. If $y \notin L_{\sigma}$ for any $\sigma$, then the worst possible scenario is that $y$ belongs to $F_{i} \cup K_{i}$ for some $i$ and $2 x-y$ belongs to $F_{j} \cup K_{j}$ for some $j$. Here, $i$ and $j$ will be bigger than $n$ because each of 
$y$ and $2 x-y$ belong to $V_{n}$. Let us look at

$$
\begin{aligned}
\frac{|f(y)+f(2 x-y)-2 f(x)|}{2|x-y|} & \leq \frac{|f(y)-f(x)|}{2|x-y|}+\frac{|f(2 x-y)-f(x)|}{2|x-y|} \\
& =\frac{f(y)}{2|x-y|}+\frac{f(2 x-y)}{2|x-y|}=\frac{\varepsilon_{i}^{2}}{2|x-y|}+\frac{\varepsilon_{j}^{2}}{2|x-y|} .
\end{aligned}
$$

Note that $|x-y|=|2 x-y-x|>\min \left\{\varepsilon_{i}, \varepsilon_{j}\right\}$ because $x \in A \subseteq C_{1}$, $y \in F_{i} \cup K_{i}$, and $2 x-y \in F_{j} \cup K_{j}$. Utilizing this observation in the above inequalities, we have that

$$
\frac{|f(y)+f(2 x-y)-2 f(x)|}{2|x-y|}<\frac{\varepsilon_{i}^{2}}{2 \varepsilon_{i}}+\frac{\varepsilon_{j}^{2}}{2 \varepsilon_{j}}<\frac{\varepsilon_{i}+\varepsilon_{j}}{2}<\varepsilon .
$$

We now want to show that $f$ is not symmetric at any point of $R \backslash A$. First, we consider the case where $p \in\left(\bigcup L_{\sigma}\right)$. We have not changed $h$ on the $L_{\sigma}$ 's. Although we have changed $h$ on the $E_{n}$ 's, the values of $f$ on the $E_{n}$ 's is bounded away from the values of $f$ on the $L_{\sigma}$ 's because $f$ only takes integer values bigger than 1 or less than -1 on the $L_{\sigma}$ 's and $f$ only takes values between 0 and $1 / 9$ on the $E_{n}$ 's. By an argument similar to the earlier one, it follows that $f$ is not symmetric at $p$. The next case we consider is that of $p \in \bigcup F_{j}$. Since $p \in F_{i}$ for some $i$, there is a sequence $\left\{p_{n}\right\} \rightarrow p$ such that, for each $n, p_{n} \in K_{i}$ and $2 p-p_{n} \notin F_{i} \cup K_{i}$. Then,

$$
\limsup _{n \rightarrow \infty}\left|f\left(2 p-p_{n}\right)+f\left(p_{n}\right)-2 f(p)\right|>0
$$

because $f\left(p_{n}\right)=f(p)=\varepsilon_{i}^{2}$ and $f\left(2 p-p_{n}\right)$ is bounded away from $\varepsilon_{i}^{2}$ (as $\left.2 p-p_{n} \notin F_{i} \cup K_{i}\right)$. Therefore, $f$ is not symmetric at $p$. We now consider the final case where $p \in R \backslash\left[\left(\bigcup F_{j}\right) \cup\left(\bigcup L_{\sigma}\right)\right]$. Since $p \notin \bigcup F_{j}, h$ is not symmetric at $p$. Therefore, $h(p)=0$, and there is a sequence $\left\{p_{n}\right\} \rightarrow p$ such that

$$
\limsup _{n \rightarrow \infty}\left|h\left(2 p-p_{n}\right)+h\left(p_{n}\right)-2 h(p)\right|>0 \text {. }
$$

Since $h$ only takes on integer values bigger than 1 or less than -1 on the $L_{\sigma}$ 's, there are infinitely many $n$ 's for which one of $2 p-p_{n}$ or $p_{n}$ belong to some $L_{\sigma}$. Since $f$ is the same as $h$ on the $L_{\sigma}$ 's and the rest takes on only the values between 0 and $1 / 9$, we also have that

$$
\limsup _{n \rightarrow \infty}\left|f\left(2 p-p_{n}\right)+f\left(p_{n}\right)-2 f(p)\right|>0 .
$$

Therefore, $f$ is not symmetric at $p$.

Going through arguments similar to the above, the reader can verify that $\mathrm{SD}(|f|)=A$ and $|f|$ is not symmetrically continuous anywhere else.

That $f$ and $|f|$ are Lebesgue measurable and have the Baire property follows from the fact that $f$ is zero on the complement of the measure zero and first category set $G \cup\left[\bigcup_{n=1}^{\infty}\left(F_{n} \cup K_{n}\right)\right]$.

We now turn to the proofs of the lemmas.

Proof of Lemma 1. We will prove this lemma by inducting on the length of $\sigma$ and $\tau$. If $|\sigma|=|\tau|=1$, then the lemma is certainly true because one of $\sigma$ or $\tau$ is 0 while the other one is 1 and $L_{0} \equiv\left[2 A-L_{\varnothing}\right] \backslash L_{\varnothing}$ and $L_{1} \equiv$ $\left[2 L_{\varnothing}-E_{1}\right] \backslash L_{\varnothing}$. Now assume that for all sequences of length $n$, the lemma is 
true. Let $|\sigma|=|\tau|=n+1$. If the last terms of both $\sigma$ and $\tau$ are zero, then there is an integer $i$ that satisfies the conclusion of the lemma with respect to $\sigma \mid n$ and $\tau \mid n$. Since the last terms of both $\sigma$ and $\tau$ are zero,

$$
L_{\sigma} \equiv\left[2 A-L_{\sigma \mid n}\right] \backslash\left(\bigcup_{\psi:|\psi| \leq n} L_{\psi}\right) \quad \text { and } \quad L_{\tau} \equiv\left[2 A-L_{\tau \mid n}\right] \backslash\left(\bigcup_{\psi:|\psi| \leq n} L_{\psi}\right) \text {. }
$$

Therefore, this integer $i$ also satisfies the conclusion of the lemma with respect to $\sigma$ and $\tau$. Now let us assume that the last terms of both $\sigma$ and $\tau$ are not zero. Without loss of generality, assume that the last term of $\sigma$ is 1 . Then, by the definition, $L_{\sigma}=\left[2 L_{\sigma \mid n}-E_{j}\right] \backslash\left(\bigcup_{\psi:|\psi| \leq n} L_{\psi}\right)$ where $j$ is the least positive integer which has not been used in the construction of any of the previous $L$ 's. If the last term of $\tau$ is zero then $L_{\tau} \equiv\left[2 A-L_{\tau \mid n}\right] \backslash\left(\bigcup_{\sigma:|\psi| \leq n} L_{\psi}\right)$, and if the last term of $\tau$ is 1 then $L_{\tau} \equiv\left[2 L_{\tau \mid n}-E_{k}\right] \backslash\left(\bigcup_{\sigma:|\psi| \leq n} L_{\psi}\right)$ where $k \neq j$ because of the way the $L$ 's are defined. In either case, $E_{j}$ does not appear in the representations of any elements of $L_{\tau}$. Therefore, we have that $E_{j}$ appears in representations of every element of $L_{\sigma}$, but $E_{j}$ does not appear in representations of any element of $L_{\tau}$, and the proof of the lemma is complete.

Proof of Lemma 2. Let $x \in A, y \in L_{\sigma}$, and $|\sigma|=n$. Let $\psi$ be such that $\psi|n=\sigma,| \psi \mid=n+1$, and the last term of $\psi$ is 0 . Since $L_{\psi} \equiv\left[2 A-L_{\sigma}\right] \backslash$ $\left(\bigcup_{\sigma:|\zeta| \leq n} L_{\zeta}\right), 2 x-y$ has to belong to some $L_{\tau}$.

If we have that $|\sigma| \neq|\tau|$, then the lemma follows easily. If $|\tau|<|\sigma|$, then let $\pi$ be a 1-term extension of $\tau$ whose last term is 0 . Then $y \in L_{\pi}=$ $\left[2 A-L_{\tau}\right] \backslash\left(\bigcup_{\sigma:|\zeta| \leq n} L_{\zeta}\right)$ because $2 x-y \in L_{\tau}$, and $x \in A$. Since all the $L$ 's are disjoint, $\pi=\sigma$. If $|\sigma|<|\tau|$, then we would take $\pi$ to be an extension of $\sigma$ whose last term is zero and get that $\pi=\tau$. The lemma follows in either case.

To complete the proof of the lemma we need to show that $|\sigma| \neq|\tau|$. If $\sigma \neq \tau$ and $|\sigma|=|\tau|$, then by Lemma 1 , there is an integer $i$ such that one of $y$ and $2 x-y$ contains an element of $E_{i}$ while the other one does not. Since $A$ and $E_{i}$ are disjoint and each element of the $L$ 's has a unique representation in terms of $C$, the element of $E_{i}$ which appears in the representation of $y$ or $2 x-y$ cannot cancel out with $x$. Thus, both $y$ and $2 x-y$ contain an element of $E_{i}$, contradicting Lemma 1 . Therefore, we cannot have $\sigma \neq \tau$ and $|\sigma|=|\tau|$.

Now what is left to show is that $\sigma \neq \tau$. First, note that $\sigma$ cannot be a zero sequence because if $\sigma$ was a zero sequence then every element of $L_{\sigma}$ can be written as $\sum_{i=0}^{n-1}(-1)^{i} a_{i}+(-1)^{n} b$ where $n=|\sigma|, a_{i} \in A, b \in B$, and none of the positive terms cancel out with the negative terms. This implies that $y$ and $2 x-y$ cannot both be in $L_{\sigma}$ because the linear representation of one of them has one more element of $C$ than the other one does. So let us assume that $\sigma$ is a nonzero sequence. Then $L_{\sigma}$ has to contain some element of $E_{i}$. A simple induction shows that if $w, z \in L_{\sigma}$ and $c_{1} b_{1}+c_{2} b_{2}+\cdots+c_{n} b_{n}+c e_{w}$ is the unique linear representation of $w$ where $e_{w}$ is the only element of $E_{i}$ and $d_{1} a_{1}+d_{2} a_{2}+\cdots+d_{m} a_{m}+d e_{z}$ is the unique linear representation of $z$ where $e_{z}$ is the only element of $E_{i}$ in the representation of $z$, then $c=d$. If $x$ and $2 x-y$ both belong to $L_{\sigma}$, then the coefficient of $E_{i}$ in the linear representation of $x$ and the coefficient of $E_{i}$ in the linear representation of $y$ 
would have different signs, contradicting the above claim. Therefore, we cannot have $\sigma=\tau$, and the proof of the lemma is complete.

Proof of Lemma 3. We will construct our set $K$ by transfinite induction. Let $\left\{p_{\sigma}\right\}_{\alpha<c}$ be a well-ordering of $M$. Since $M$ is nowhere dense and $N$ is $c$-dense in $U$, we have that, for every $x \in M$, there is $y \in N$, arbitrarily close to $x$, such that $2 x-y \notin M$. Using this observation, pick an increasing sequence $\left\{p_{0, n}\right\}_{n=1}^{\infty} \rightarrow p_{0}$ such that, for every $n, 2 p_{0}-p_{0, n}$ does not belong to $M$. Suppose, for each $\alpha<\beta,\left\{p_{\alpha, n}\right\}_{n=1}^{\infty}$ has been picked. Let $T=\left\{2 p_{\zeta}-p_{\alpha, n}: \zeta \leq\right.$ $\beta, \alpha<\beta$, and $n$ is a positive integer $\}$. Choose $\left\{p_{\beta, n}\right\}_{n=1}^{\infty}$ to be an increasing sequence which converges to $p_{\beta}$ such that, for every integer $n, p_{\beta, n} \in N \backslash T$ and $2 p_{\beta}-p_{\beta, n} \notin M$. It is possible to pick $\left\{p_{\beta, n}\right\}_{n=1}^{\infty}$ in this fashion because the cardinality of the set $T$ is less than $c$, which implies that $N \backslash T$ is still $c$-dense in $U$.

Let $K=\left\{p_{\alpha, n}: \alpha<c\right.$ and $n$ is a positive integer $\}$. Now we want to show that $K$ satisfies the conclusion of our theorem. Let $x \in M$. There is $\delta$ such that $x=p_{\delta}$. The sequence $\left\{p_{\delta, n}\right\}_{n=1}^{\infty} \rightarrow p_{\delta}$. By the method in which $p_{\delta, n}$ was picked, we have that $2 p_{\delta}-p_{\delta, n} \notin M$. What we now need to show is that $2 p_{\delta}-p_{\delta, n} \notin K$. To obtain a contradiction, assume that $2 p_{\delta}-p_{\delta, n} \in K$. Then $2 p_{\delta}-p_{\delta, n}=p_{\gamma, m}$ for some ordinal $\gamma$ and integer $m$. Note that $\gamma \neq \delta$ because $\left\{p_{\delta, n}\right\}_{n=1}^{\infty}$ is an increasing sequence which converges to $p_{\delta}$. Therefore, $\gamma<\delta$ or $\delta<\gamma$. If $\gamma<\delta$ then $p_{\delta, n} \notin\left\{2 p_{\zeta}-p_{\alpha, n}: \zeta \leq \delta, \alpha<\delta\right.$, and $n$ is a positive integer $\}$, which implies that $p_{\delta, n} \neq 2 p_{\delta}-p_{\gamma, m}$, contradicting the fact that $2 p_{\delta}-p_{\delta, n}=p_{\gamma, m}$. If $\delta<\gamma$, then $p_{\gamma, m} \notin\left\{2 p_{\zeta}-p_{\alpha, n}: \zeta \leq \gamma, \alpha<\gamma\right.$, and $n$ is a positive integer $\}$, which implies that $p_{\gamma, m} \neq 2 p_{\delta}-p_{\delta, n}$ and leads to a contradiction. Therefore, $2 p_{\delta}-p_{\delta, n} \notin K$, and the proof of the lemma is complete.

\section{REFERENCES}

1. U. B. Darji, Symmetric functions, Lebesgue measurability and the Baire property, Proc. Amer. Math. Soc. (to appear).

2. P. Erdös and A. H. Stone, On the sum of two Borel sets, Proc. Amer. Math. Soc. 25 (1970), 304-306.

3. P. Humke and M. Laczkovich, $A$ historical note on the measurability properties of symmetrically continuous and symmetrically differentiable functions, Real Analysis Exchange 15 (1989-1990), 768-771.

4. F. B. Jones, Measure and other properties of a Hamel basis, Bull. Amer. Math. Soc. 48 (1942), 472-481.

5. S. Marcus, Les ensembles $F_{\sigma}$ et la continuite symetrique, Acad. Roy. P. Romine. Bull. Sti. Sect. Sti. Mat. Fiz. 7 (1955), 871-886.

6. J. Mycielski, Independent sets in topological algebras, Fund. Math. 55 (1964), 139-147.

7. J. von Neumann, Ein System algebraisch unabhangiger Zahlen, Math. Ann. 99 (1928), 134-141.

8. C. A. Rogers, A linear Borel set whose difference set is not a Borel set, Bull. London Math. Soc. 2 (1970), 41-42.

Department of Mathematics, North Carolina State University, Raleigh, North CarOLINA 27695-8205

E-mail address: udarji@guest1.math.ncsu.edu 